Proyecciones

Vol. 22, No 3, pp. 161-180, December 2003.

Universidad Católica del Norte

Antofagasta - Chile

\title{
AN EXTENSION OF THE POINCARÉ COMPACTIFICATION AND A GEOMETRIC INTERPRETATION
}

\author{
CLAUDIO VIDAL \\ Universidade Federal de Pernambuco - Brasil. \\ and \\ PEDRO GÓMEZ \\ Universidad Federal de Paraiba - Brasil
}

\begin{abstract}
Our purpose in this paper is to understand the geometry of the Poincaré compactification and to apply this technique to prove that there exists a Poincaré compactification of vector fields defined by rational functions and of vector field that are the quotient of some power of polynomial. We will give also a global expressions for the Poincaré vector field associated. Furthermore, we summarize these results proving that there exist a Poincaré vector field for any vector field whose rate of growth at infinity of each component is not bigger than a polynomial growth.
\end{abstract}

Mathematics Subject Classification : 34A34, 34C05, 34A99.

Key Words : Poincaré compactification, rational vector field, equilibrium solutions. 


\section{Introduction}

The study of solutions escaping to infinity has been an important tool in order to understand the global picture of a dynamical system in $\mathbb{R}^{n}$. The compactification technique consists in writing the equations of motion as a vector field and then applying the Poincaré compactification, which is a method to extend analytically the vector field to a compact manifold, in fact to a sphere. This tool or method is very important to study the qualitative dynamic of the flow at infinity or in the unbounded part.

The main idea of this method is to identify $\mathbb{R}^{n}$ with northern and southern hemispheres through simple projections, then the vector field $X$ on $\mathbb{R}^{n}$ can be extended to a vector field $\tilde{X}$ on $S^{n}$; this method is called the Poincaré compactification.

In [10] dated 1881, Poincaré began the study of polynomial vector fields on the plane $\mathbb{R}^{2}$, by means of central projection of the paths on a sphere $S^{2}$, tangent to the plane at the origin. Thus, he provided the means for studying the behavior of the field on a neighborhood of infinity, which is represented by the equator, $S^{1}$. In different papers González [8] and Cima and Llibre [2] showed that any polynomial vector field on $\mathbb{R}^{n}$ can be extended analytically to the $n$-dimensional sphere. Using these ideas, in the papers [5], [6], [7] the authors studied the Poincaré compactification for homogeneous vector fields, in particular for homogeneous polynomial vector fields and by homogeneous polynomial Hamiltonian vector field. Then, in each case they give a global expressions for the Poincaré compactification. As an application, and using the fact that the vector field of the $n$-body problem can always be written in the form of a polynomial vector field (see [9] for example), the Poincaré compactifications for the Kepler problem on the line and on the plane and for the collinear 3-body problem are computed. The main disadvantage here for obtaining this polynomial vector field is the use (in general) of redundant variables.

Our purpose in this paper is to understand the geometry of the Poincaré compactification (see the second section) and to apply this technique to prove that there exists a Poincaré compactification of vector fields defined by rational functions and of vector field that are the quotient of some power of polynomial. We will also give a global expressions for the Poincaré vector field associated. These results are proved in the third section. In the fourth section, we show that the main requirement for constructing the Poincare compactification of some vector field $X$ is the fact that the rate of growth at infinity of each component of the field $X$ must be not bigger 
than a polynomial growth, i.e., there exist some polynomial vector field $Y=\left(P_{1}, \ldots, P_{n}\right)\left(n_{j}\right.$ is the degree of $\left.P_{j}\right)$ such that

$$
\lim _{\|\mathbf{x}\| \rightarrow+\infty} \frac{f_{j}(\mathbf{x})}{P_{j}(\mathbf{x})}=c_{j}, \quad j=1, \ldots, n
$$

where $c_{j}$ are some constants. In this case we prove that this property on $X$ implies that it is possible to get the Poincaré vector field $P(X)$.

In the fifth section, we study the behavior of the induced vector field at infinity in the 2-dimensional case and also we study some generic properties, namely, generically the equilibrium solutions at the infinity are hyperbolic.

Finally, in the sixth section, we apply this method to study some restricted three-body problems in Celestial Mechanics, but, as we apply this result directly to rational vector fields, in general we do not introduce redundant variables. The knowledge of the final configurations and velocities (escaping solutions in the phase space) aim to give a mechanical explanation to observed phenomena in Celestial Mechanics as is the case of the age and formation of planetary systems, binary stars, comets, etc. Chazy in [1] gave the first general classification in the 3-body problem when one or several of the mutual distances go to infinity. In particular he proved that there exist a great diversity of interesting motions in this particular case of the n-body problem.

\section{Poincaré's compactification method and a geometric inter- pretation}

Let

$$
X(\mathbf{x})=\left(f_{1}(\mathbf{x}), \ldots, f_{n}(\mathbf{x})\right),
$$

be a vector field on $\mathbb{R}^{n}$, a noncompact manifold, where $f_{i}$ are functions of $C^{1}$ class in $\mathbb{R}^{n}$. First, we identify $\mathbb{R}^{n}$ with the hyperplane

$$
\Pi=\left\{\mathbf{x} \in \mathbb{R}^{n+1} / x_{n+1}=0\right\}
$$

in $\mathbb{R}^{n+1}$ which is tangent to the Poincaré's sphere $S^{n}=\left\{\mathbf{y} \in \mathbb{R}^{n+1} /\|\mathbf{y}\|=\right.$ $1\}$ in $\mathbb{R}^{n+1}$ at the north pole. Then, we take the central projection from the sphere $S^{n}$ to the hyperplane $\Pi$, that is, for each point in $\Pi$ we draw the straight line through this point and the origin in $\mathbb{R}^{n+1}$, obtaining in this way two antipodal points in $S^{n}$, one in the open northern hemisphere $H^{+}$ and the other in the open southern hemisphere $H^{-}$of $S^{n}$. More concretely, this construction defines the following two diffeomorphism

$$
\Phi^{+}: \mathbb{R}^{n} \rightarrow H^{+}, \quad \text { and } \quad \Phi^{-}: \mathbb{R}^{n} \rightarrow H^{-}
$$


given by,

$$
\begin{aligned}
& \Phi^{+}(\mathbf{x})=\frac{1}{\Delta(\mathbf{x})}\left(x_{1}, \ldots, x_{n}, 1\right) \\
& \Phi^{-}(\mathbf{x})=-\frac{1}{\Delta(\mathbf{x})}\left(x_{1}, \ldots, x_{n}, 1\right)
\end{aligned}
$$

where $\Delta(\mathbf{x})=\left(1+\|\mathbf{x}\|^{2}\right)^{1 / 2}$.

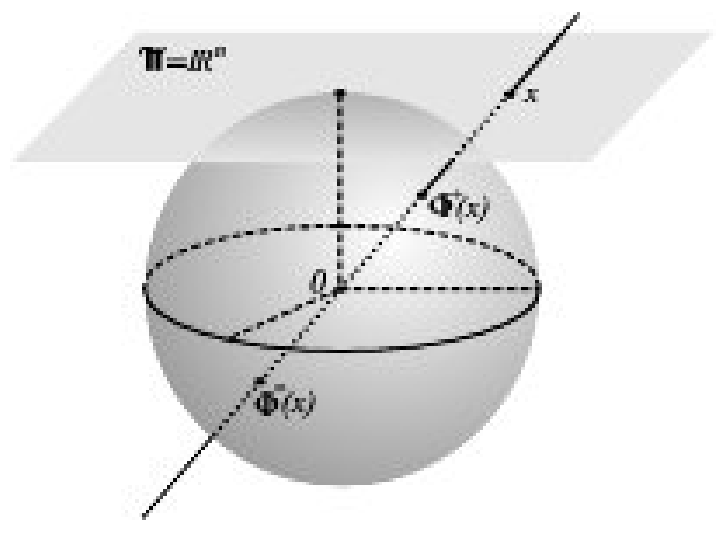

Figure 1 : The central projections.

In this form the vector field $X$ induces a vector field $\bar{X}$ on $H^{+} \cup H^{-}$defined by

$$
\bar{X}(\mathbf{y})=\left\{\begin{array}{lll}
D \Phi^{+}(\mathbf{x}) X(\mathbf{x}), & \text { if } & \mathbf{y}=\Phi^{+}(\mathbf{x}) \\
D \Phi^{-}(\mathbf{x}) X(\mathbf{x}), & \text { if } & \mathbf{y}=\Phi^{-}(\mathbf{x}) .
\end{array}\right.
$$

Therefore,

$$
\begin{aligned}
\bar{X}(\mathbf{y})= & D \Phi^{ \pm}(\mathbf{x}) X(\mathbf{x}) \\
= & \frac{d}{d t}\left(\Phi^{ \pm}(\varphi(t, \mathbf{x}))\right)_{t=0} \\
= & \frac{d}{d t}\left( \pm \frac{1}{\Delta(\varphi(t, \mathbf{x}))}(\varphi(t, \mathbf{x}), 1)\right)_{t=0} \\
= & \left. \pm\left[\frac{1}{\Delta(\mathbf{x})} X(\mathbf{x}), 0\right)-\frac{X(\mathbf{x}, 0) \cdot(\mathbf{x}, 1)}{\Delta(\mathbf{x})^{3}}(\mathbf{x}, 1)\right] \\
= & \pm \frac{1}{\Delta(\mathbf{x})}\left[(X(\mathbf{x}), 0)-\operatorname{Proj}_{y}(X(\mathbf{x}, 0))\right] \\
= & y_{n+1} \operatorname{Proj}_{y}(X(\mathbf{x}), 0) \\
= & y_{n+1}\left(f_{1}(\mathbf{x})-\left[y_{1} f_{1}(\mathbf{x})+\ldots+y_{n} f_{n}(\mathbf{x})\right] y_{1}, \ldots,\right. \\
& f_{n}(\mathbf{x})-\left[y_{1} f_{1}(\mathbf{x})+\ldots+y_{n} f_{n}(\mathbf{x})\right] y_{n},-\left[y_{1} f_{1}(\mathbf{x})+\ldots+y_{n} f_{n}(\mathbf{x})\right] \\
& \left.y_{n+1}\right)
\end{aligned}
$$


where $\Phi^{ \pm}(\mathbf{x})=\mathbf{y}, \varphi(t, \mathbf{x})$ is the integral curve associated to the vector field $X$ satisfying the initial condition $\varphi(0, \mathbf{x})=\mathbf{x}$ and $\operatorname{Proj}_{w}(\mathbf{v})$ means the orthogonal projection of the vector $\mathbf{v}$ on the subspace $W$. Note that by (2.2) we have

$$
\mathbf{x}=\left\{\begin{array}{l}
\left(\frac{y_{1}}{y_{n+1}}, \ldots, \frac{y_{n}}{y_{n+1}}\right), \quad \text { if } \quad \mathbf{y}=\Phi^{+}(\mathbf{x}) \\
\left(-\frac{y_{1}}{y_{n+1}}, \ldots,-\frac{y_{n}}{y_{n+1}}\right), \quad \text { if } \quad \mathbf{y}=\Phi^{-}(\mathbf{x}),
\end{array}\right.
$$

so, the function $f_{j}$ in (2.4) must satisfy:

$$
f_{j}(\mathbf{x})=\left\{\begin{array}{l}
f_{j}\left(\frac{y_{1}}{y_{n+1}}, \ldots, \frac{y_{n}}{y_{n+1}}\right), \quad \text { if } \quad \mathbf{y}=\Phi^{+}(\mathbf{x}) \\
f_{j}\left(-\frac{y_{1}}{y_{n+1}}, \ldots,-\frac{y_{n}}{y_{n+1}}\right), \quad \text { if } \quad \mathbf{y}=\Phi^{-}(\mathbf{x}) .
\end{array}\right.
$$

Lemma 1. Under central projection every straight line in the plane $\Pi$ is mapped on great circle of the sphere $S^{n}$.

Proof: In fact, the proof is clear because the great circles on the sphere $S^{n}$ are by definition the intersection of the sphere $S^{n}$ and a plane, $P$, through the center $O$ of the sphere. Then, by definition of the map $\Phi^{+}$we have

$$
\Phi^{+}(l) \subset P \cap S^{n}
$$

for every straight line $l$ in the plane $\Pi$.

If we are interested in studying the flow associated to the vector field $X$ at the infinity, i.e., $\Delta(\mathbf{x})=+\infty$, this problem in the new variables $\mathbf{y}$ over the sphere $S^{n}$, by means of the change of coordinates given in (2.2), corresponds to $y_{n+1}=0$, i.e., it is necessary to study the new vector field $\bar{X}$, defined in (2.3), on the equator of $S^{n}$, i.e., on $S^{n-1}$. However, in general the vector field $\bar{X}$ is not well defined on the equator and also it is not invariant on the equator $y_{n+1}=0$. Therefore, depending on the vector field $X$, sometimes it is necessary to modify the vector field $\bar{X}$ by an appropriate scalar function such that the new vector field is now well defined and invariant on the equator $y_{n+1}=0$. This method is called Poincaré compactification of $X$ and it will be denoted by $P(X)$; we will refer to $P(X)$ as the Poincaré vector field. 
Let $l$ be the straight line in the plane $\Pi$ through the origin, i.e, $l: t \mathbf{v}$ where $\mathbf{v} \in \mathbb{R}^{n}$ and $\|\mathbf{v}\|=1$, then

$$
\begin{aligned}
& \Phi^{+}(t \mathbf{v})=\frac{1}{\sqrt{1+t^{2}}}\left(t v_{1}, \ldots, t v_{n}, 1\right), \\
& \Phi^{-}(t \mathbf{v})=-\frac{1}{\sqrt{1+t^{2}}}\left(t v_{1}, \ldots, t v_{n}, 1\right) .
\end{aligned}
$$

Supposing that

$$
\Phi^{+}(t \mathbf{v}) \rightarrow \pm(\mathbf{v}, 0) \quad \text { as } \quad t \rightarrow \pm \infty
$$

then

$$
\Phi^{-}(t \mathbf{v}) \rightarrow \mp(\mathbf{v}, 0) \quad \text { as } \quad t \rightarrow \pm \infty .
$$

So, we have the following criterium of continuity.

Lemma 2. (Continuity condition) A necessary condition for the Poincaré compactification to exist is that for every straight line $l$ in the plane $\Pi$ there is a function $\lambda: S^{n} \rightarrow \mathbb{R}$ such that the following limits

$$
\begin{aligned}
& \lim _{t \rightarrow \pm \infty} \lambda(t \mathbf{v}) y_{n+1} \bar{X}\left(\Phi^{+}(t \mathbf{v})\right) \\
& \lim _{t \rightarrow \mp \infty} \lambda(t \mathbf{v}) y_{n+1} \bar{X}\left(\Phi^{-}(t \mathbf{v})\right)
\end{aligned}
$$

there exist and both agree.

\section{Poincaré compactification for rational vector field and quo- tient of power of polynomial vector field}

Firstly, we will consider the case where $f_{j}$ in (2.1) is a rational function, which we denote by

$$
f_{j}(\mathbf{x})=\frac{P_{j}(\mathbf{x})}{Q_{j}(\mathbf{x})}, \quad j=1, \ldots, n
$$

where the degree of $P_{j}$ is $n_{j}$ and the degree of $Q_{j}$ is $m_{j}$. Letting, $m=$ $\max \left\{n_{j}, m_{j} j=1, \ldots, n\right\}$ we have

Theorem 1. The induced vector field $\bar{X}$ on $S^{n} \backslash S^{n-1}$, defined in (2.3), from the vector field $X$ given in (2.1), by means of the differentials of $\Phi^{+}$and $\Phi^{-}$with $f_{j}$ given by (3.1), can be extended to the whole of the sphere after multiplication by $y_{n+1}^{m-1}$, and in such a way that the equator is invariant. 
Proof: It is sufficient to analyze the term $f_{j}(\mathbf{y})$. Let

$$
\begin{aligned}
P_{j}(\mathbf{x}) & =P_{j}^{(0)}+P_{j}^{(1)}(\mathbf{x})+\ldots+P_{j}^{\left(n_{j}\right)}(\mathbf{x}), \\
Q_{j}(\mathbf{x}) & =Q_{j}^{(0)}+Q_{j}^{(1)}(\mathbf{x})+\ldots+Q_{j}^{\left(m_{j}\right)}(\mathbf{x})
\end{aligned}
$$

where $P_{j}^{(s)}$ and $Q_{j}^{(s)}$ are homogeneous polynomials of degree $s$ in the variables $\mathbf{x}$. By analogy, we will only analyze the case $\mathbf{y}=\Phi^{+}(\mathbf{x})$. Thus,

$$
\begin{aligned}
& P_{j}^{(s)}(\mathbf{x})=P_{j}^{(s)}\left(\frac{y_{1}}{y_{n+1}}, \ldots, \frac{y_{n}}{y_{n+1}}\right)=\frac{1}{y_{n+1}^{s}} P_{j}^{(s)}\left(y_{1}, \ldots, y_{n}\right) \\
& Q_{j}^{(s)}(\mathbf{x})=Q_{j}^{(s)}\left(\frac{y_{1}}{y_{n+1}}, \ldots, \frac{y_{n}}{y_{n+1}}\right)=\frac{1}{y_{n+1}^{s}} Q_{j}^{(s)}\left(y_{1}, \ldots, y_{n}\right),
\end{aligned}
$$

and then,

$$
\begin{aligned}
P_{j}\left(\frac{y_{1}}{y_{n+1}}, \ldots, \frac{y_{n}}{y_{n+1}}\right)= & \frac{1}{y_{n+1}^{n_{j}}}\left[y_{n+1}^{n_{j}} P_{j}^{(0)}+\ldots+y_{n+1} P_{j}^{\left(n_{j}-1\right)}\left(y_{1}, \ldots, y_{n}\right)\right. \\
& \left.+P_{j}^{\left(n_{j}\right)}\left(y_{1}, \ldots, y_{n}\right)\right], \\
Q_{j}\left(\frac{y_{1}}{y_{n+1}}, \ldots, \frac{y_{n}}{y_{n+1}}\right)= & \frac{1}{y_{n+1}}\left[y_{n+1}^{m_{j}} Q_{j}^{(0)}+\ldots+y_{n+1} Q_{j}^{\left(m_{j}-1\right)}\left(y_{1}, \ldots, y_{n}\right)\right. \\
& \left.+Q_{j}^{\left(m_{j}\right)}\left(y_{1}, \ldots, y_{n}\right)\right] .
\end{aligned}
$$

Therefore, after multiplication by the factor $y_{n+1}^{m-1}$ in each component of the vector field $\bar{X}$, we obtain an extension of the vector field $\bar{X}$ on the equator $y_{n+1}=0$, which is invariant.

Remarks. The vector field $P(X)$ on the equator $S^{n-1}$ is given by:

$$
P(X)\left(y_{1}, \ldots, y_{n}\right)=\left(P_{1}(X)\left(y_{1}, \ldots, y_{n}\right), \ldots, P_{n}(X)\left(y_{1}, \ldots, y_{n}\right)\right)
$$

where we will consider $\mathbf{y}=\Phi^{+}(\mathbf{x})$ (the other case is analogous) and for $j=1, \ldots, n$

$$
\begin{aligned}
P_{j}(X)\left(y_{1}, \ldots, y_{n}\right) & =y_{n+1}^{m-1}\left[f_{j}(\mathbf{x})-y_{j} \sum_{s=1}^{n} y_{s} f_{s}(\mathbf{x})\right] \\
& =\delta_{j} \frac{P_{j}^{\left(n_{j}\right)}\left(y_{1}, \ldots, y_{n}\right)}{Q_{j}^{\left(m_{j}\right)}\left(y_{1}, \ldots, y_{n}\right)}-y_{j}\left[\sum_{s=1}^{n} \delta_{s} \frac{P_{j}^{\left(n_{s}\right)}\left(y_{1}, \ldots, y_{n}\right)}{Q_{j}^{\left(m_{s}\right)}\left(y_{1}, \ldots, y_{n}\right)}\right]
\end{aligned}
$$

where

$$
\delta_{s}= \begin{cases}1, & \text { if } n_{s}-m_{s}=m \\ 0, & \text { otherwise }\end{cases}
$$


Another very important remark is the smoothness of the compactified vector field $P(X)$. In fact, we have immediately that this vector field will be analytic on the set $\left\{\left(y_{1}, \ldots, y_{n}\right) \in S^{n-1} / Q_{j}^{\left(m_{s}\right)}\left(y_{1}, \ldots, y_{n}\right) \neq 0\right\}$.

In the second case, we will assume that $f_{j}$, given in (2.1), is a function such that

$$
f_{j}(\mathbf{x})=\frac{\left[P_{j}(\mathbf{x})\right]^{\alpha_{j}}}{\left[Q_{j}(\mathbf{x})\right]^{\beta_{j}}}, \quad j=1, \ldots, n .
$$

As before, $P_{j}$ and $Q_{j}$ are polynomials, where $\alpha_{j}$ and $\beta_{j}$ are real non-zero constant, and $f_{j}(\mathbf{x})$ is well defined.

Therefore following the same arguments as in Theorem 1, but defining

$$
m=\max \left\{\alpha_{j} n_{j}-\beta_{j} m_{j} / j=1, \ldots, n\right\},
$$

in this case we have.

Theorem 2. The induced vector field $\bar{X}$ on $S^{n} \backslash S^{n-1}$, defined in (2.3), from the vector field $X$ given in (2.1), by means of the differentials of $\Phi^{+}$and $\Phi^{-}$with $f_{j}$ given by (3.1), can be extended to the whole of the sphere after multiplication by $y_{n+1}^{m-1}$, and in such a way that the equator is invariant.

Remarks. In this case the vector field $P(X)$ on the equator $S^{n-1}$ is given by:

$$
P(X)\left(y_{1}, \ldots, y_{n}\right)=\left(P_{1}(X)\left(y_{1}, \ldots, y_{n}\right), \ldots, P_{n}(X)\left(y_{1}, \ldots, y_{n}\right)\right)
$$

where we will consider $\mathbf{y}=\Phi^{+}(\mathbf{x})$ (the other case is analogous) and for $j=1, \ldots, n$

$$
\begin{aligned}
P_{j}(X)\left(y_{1}, \ldots, y_{n}\right) & =y_{n+1}^{m-1}\left[f_{j}(\mathbf{x})-y_{j} \sum_{s=1}^{n} y_{s} f_{s}(\mathbf{x})\right] \\
& =\delta_{j} \frac{\left[P_{j}^{\left(n_{j}\right)}\left(y_{1}, \ldots, y_{n}\right)\right]^{\alpha_{j}}}{\left[Q_{j}^{\left(m_{j}\right)}\left(y_{1}, \ldots, y_{n}\right)\right]^{\beta_{j}}}-y_{j}\left[\sum_{s=1}^{n} \delta_{s} \frac{\left[P_{j}^{\left(n_{s}\right)}\left(y_{1}, \ldots, y_{n}\right)\right]^{\alpha_{s}}}{\left[Q_{j}^{\left(m_{s}\right)}\left(y_{1}, \ldots, y_{n}\right)\right]^{\alpha_{s}}}\right]
\end{aligned}
$$

where

$$
\delta_{s}= \begin{cases}1, & \text { if } \alpha_{s} n_{s}-\beta_{s} m_{s}=m \\ 0, & \text { otherwise }\end{cases}
$$


It follows immediately that the degree of differentiability of the vector field $P(X)$ will depend on $\alpha_{j}, \beta_{j}$ and the sets

$\left\{\left(y_{1}, \ldots, y_{n}\right) \in S^{n-1} / P_{j}^{\left(m_{s}\right)}\left(y_{1}, \ldots, y_{n}\right) \neq 0\right\}$ and

$\left\{\left(y_{1}, \ldots, y_{n}\right) \in S^{n-1} / Q_{j}^{\left(m_{s}\right)}\left(y_{1}, \ldots, y_{n}\right) \neq 0\right\}$. So, the differentiability must be analyzed separately in each case.

Corollary 1. Let

$$
X=X_{H}+X_{F}
$$

where $X_{H}=\left(g_{1}, \ldots, g_{n}\right)$ is a vector field defined by polynomials $g_{j}$ and $X_{F}=\left(f_{1}, \ldots, f_{n}\right)$ is the vector field defined in (3.7). Let $k_{j}$ be the degree of $g_{j}$ and $m=\max \left\{\alpha_{j} n_{j}-\beta_{j} m_{j}, k_{j} / j=1, \ldots, n\right\}$. Then, the induced vector field $\bar{X}$ given in (2.3) from the vector field $X$ given in (2.1) by means of the differentials of $\Phi^{+}$and $\Phi^{-}$can be extended to the whole of the sphere after multiplication by $y_{n+1}^{m-1}$, and in such a way that the equator is invariant.

\section{Generalization}

In the previous section we have restricted our study to the set of all rational vector fields and vector fields whose components are the quotient of power of polynomials as in (3.7). In all these cases the Poincaré compactification vector field can be obtained, and we observe that in these cases the rate of growth at infinity of each component is not bigger than a polynomial growth. We emphasize that this property implies that it is possible to get the compactified vector field $P(X)$.

Let the vector field $X$ given in (2.1) and we will assume that there is a polynomial vector field $Y=\left(P_{1}, \ldots, P_{n}\right)\left(n_{j}\right.$ is the degree of $\left.P_{j}\right)$ such that

$$
\lim _{\|\mathbf{x}\| \rightarrow+\infty} \frac{f_{j}(\mathbf{x})}{P_{j}(\mathbf{x})}=c_{j}, \quad j=1, \ldots, n
$$

with $c_{j}$ constant. Let $m=\max \left\{n_{j} / j=1, \ldots, n\right\}$. The following theorem is a generalization of the previous results. 
Theorem 3. The induced vector field $\bar{X}$ on $S^{n} \backslash S^{n-1}$, defined in (2.3), from the vector field $X$ given in (2.1), by means of the differentials of $\Phi^{+}$ and $\Phi^{-}$with $f_{j}$ given by (4.1), can be extended to the whole of the sphere after multiplication by $y_{n+1}^{m-1}$, and in such a way that the equator is left invariant.

Proof: It is sufficient to prove that there is the limit

$$
\lim _{y_{n+1} \rightarrow 0} y_{n+1}^{m} f_{j}\left(\frac{y_{1}}{y_{n+1}}, \ldots, \frac{y_{n}}{y_{n+1}}\right),
$$

for every $j=1, \ldots, n$. Let,

$$
P_{j}(\mathbf{x})=P_{j}^{(0)}+P_{j}^{(1)}(\mathbf{x})+\ldots+P_{j}^{\left(n_{j}\right)}(\mathbf{x}),
$$

where $P_{j}^{(s)}$ is an homogeneous polynomial of degree $s$ in the variable $\mathbf{x}$. We will only analyze the case $\mathbf{y}=\Phi^{+}(\mathbf{x})$ since the other case is analogous. Thus,

$$
\begin{aligned}
c_{j} & =\lim _{\|\mathbf{x}\| \rightarrow+\infty} \frac{f_{j}(\mathbf{x})}{P_{j}(\mathbf{x})} \\
& =\lim _{\|\mathbf{x}\| \rightarrow+\infty} \frac{f_{j}(\mathbf{x})}{P_{j}^{(0)}+P_{j}^{(1)}(\mathbf{x})+\ldots+P_{j}^{\left(n_{j}\right)}(\mathbf{x})} \\
& =\lim _{y_{n+1} \rightarrow 0} \frac{f_{j}\left(\frac{y_{1}}{y_{n+1}}, \ldots, \frac{y_{n}}{y_{n+1}}\right)}{y_{n+1}^{-n_{j}}\left[y_{n+1}^{n_{j}} P_{j}^{(0)}+y_{n+1}^{n_{j}-1} P_{j}^{(1)}\left(y_{1}, \ldots, y_{n}\right), \ldots+P_{j}^{\left(n_{j}\right)}\left(y_{1}, \ldots, y_{n}\right)\right.}
\end{aligned}
$$

and we have

$$
\lim _{y_{n+1} \rightarrow 0} y_{n+1}^{n_{j}} f_{j}\left(\frac{y_{1}}{y_{n+1}}, \ldots, \frac{y_{n}}{y_{n+1}}\right)=c_{j} P_{j}^{\left(n_{j}\right)}\left(y_{1}, \ldots, y_{n}\right) .
$$

Therefore, after multiplication by the factor $y_{n+1}^{m-1}$ in each component of the vector field $\bar{X}$, we obtain an extension of the vector field $\bar{X}$ on the equator $y_{n+1}=0$, which is left invariant.

\section{Behavior of the induced field at infinity for the 2-dimensional case and generic properties}

In this section, we shall investigate under which conditions there are equilibrium points or closed orbits at infinity, and of which types they are for the 2-dimensional case. 
Let us go now into analytical details. The sphere $S^{2}$ will be considered as a differentiable manifold, the corresponding coordinate maps are:

$$
\phi_{i}: U_{i} \rightarrow \mathbb{R}^{2}, \quad \text { and } \quad \psi_{i}: V_{i} \rightarrow \mathbb{R}^{2}
$$

where $U_{i}=\left\{\mathbf{y} \in S^{2} / y_{i}>0\right\}, V_{i}=\left\{\mathbf{y} \in S^{2} / y_{i}<0\right\}(i=1,2,3)$ and

$$
\phi_{i}(\mathbf{y})=\psi_{i}(\mathbf{y})=\left(\frac{y_{j}}{y_{i}}, \frac{y_{k}}{y_{i}}\right)
$$

with $i, j, k=1,2,3 ; j<k$. We shall denote by $\mathbf{z}=\left(z_{1}, z_{2}\right)$ the value of $\phi_{i}(\mathbf{y})$ or $\psi_{i}(\mathbf{y})$ for any $i$, so that $\mathbf{z}$ represents different things according to the case under consideration. In order to find the explicit expression for the induced compactified vector field on $\mathbb{R}^{2}$ for the case in which $\mathbf{y} \in U_{i}$ or $\mathbf{y} \in V_{i}(i=1,2,3)$, it is convenient to express the obtained compactified vector field (3.6) or (3.9) in terms of the variable $\mathbf{z}$. Making straightforward computations we arrive at the final expression for the field on $U_{1}$

$$
\frac{z_{2}^{m}}{\Delta(\mathbf{z})^{m-1}}\left(f_{2}\left(\frac{1}{z_{2}}, \frac{z_{1}}{z_{2}}\right)-z_{1} f_{1}\left(\frac{1}{z_{2}}, \frac{z_{1}}{z_{2}}\right),-z_{2} f_{1}\left(\frac{1}{z_{2}}, \frac{z_{1}}{z_{2}}\right)\right)
$$

analogously on $U_{2}$ we have

$$
\frac{z_{2}^{m}}{\Delta(\mathbf{z})^{m-1}}\left(f_{1}\left(\frac{z_{1}}{z_{2}}, \frac{1}{z_{2}}\right)-z_{1} f_{2}\left(\frac{z_{1}}{z_{2}}, \frac{1}{z_{2}}\right),-z_{2} f_{2}\left(\frac{z_{1}}{z_{2}}, \frac{1}{z_{2}}\right)\right)
$$

and finally on $U_{3}$

$$
\frac{z_{2}^{m}}{\Delta(\mathbf{z})^{m-1}}\left(f_{1}\left(z_{1}, z_{2}\right), f_{2}\left(z_{1}, z_{2}\right)\right)
$$

where $f_{j}$ is given by (3.1) satisfying (3.2) in the rational case and by (3.7) again satisfying (3.2) in the power rational case. For the case in which $\mathbf{y} \in V_{i}, i=1,2,3$, we obtain the same expressions (5.2) to (5.4) except for the following fact: in $V_{1}$ we have $y_{1}<0$ and then $y_{3}$ and $z_{2}$ are of different sign. Thus $y_{3}=-\frac{z_{2}}{\Delta(\mathbf{z})}$ and (5.2) must be multiplied by $(-1)^{m-1}$ to obtain the field on $V_{1}$. Also, it can be seen that (5.3) and (5.4) have to be multiplied by $(-1)^{m-1}$.

In the neighborhoods $U_{1}, U_{2}, V_{1}$ and $V_{2}$ which are the only ones containing points at infinity, our vector field is given by the expressions (5.2) and (5.3). To simplify, we will call $\left(z_{1}, z_{2}\right)$ by $(\mu, \nu)$. Now, we recall that the equator $S^{1}$ is an invariant set and the second component of (5.2) or (5.3) is zero for $\nu=\frac{1}{\Delta(\mathbf{z})}=0$. Then, $P(X)$ has equilibrium points in $S^{1}$ and one of them $(\mu, \nu=0)$ must satisfy the following equation

$$
\left.\nu^{m}\left[R_{2}\left(\frac{1}{\nu}, \frac{\mu}{\nu}\right)-\mu R_{1}\left(\frac{1}{\nu}, \frac{\mu}{\nu}\right)\right]\right|_{\nu=0}=0, \quad \text { in } \quad U_{1}
$$


and

$$
\left.\nu^{m}\left[R_{1}\left(\frac{\mu}{\nu}, \frac{1}{\nu}\right)-\mu R_{2}\left(\frac{\mu}{\nu}, \frac{1}{\nu}\right)\right]\right|_{\nu=0}=0, \quad \text { in } \quad U_{2}
$$

which are well defined with the appropriate choice of $m$.

\subsection{Equilibrium solutions for rational vector field}

We will analyze the vector field given by (3.1) satisfying (3.2) for $n=2$, i.e., the vector field $X$ given in $(2.1)$ on $\mathbb{R}^{2}$ is rational. Maintaining the preliminary notations, the equation (5.5) on $U_{1}$ is equivalent to

$$
F(\mu)=\delta_{2} \frac{p_{2}^{\left(n_{2}\right)}(1, \mu)}{q_{2}^{\left(m_{2}\right)}(1, \mu)}-\delta_{1} \mu \frac{p_{1}^{\left(n_{1}\right)}(1, \mu)}{q_{1}^{\left(m_{1}\right)}(1, \mu)}=0 .
$$

To analyze the hyperbolicity of the equilibrium solutions it is necessary to calculate the variational equations of the vector field (5.2) through the equilibrium solution $\left(\mu^{*}, 0\right)$. In this case the linear part is given by

$$
A=\left(\begin{array}{cc}
\frac{d F\left(\mu^{*}\right)}{d \mu} & \star \\
0 & -\left[m+1-\left(n_{1}-m_{1}\right)\right] \delta_{1} \frac{p_{1}^{\left(n_{1}\right)}\left(1, \mu^{*}\right)}{q_{1}^{\left(m_{1}\right)}\left(1, \mu^{*}\right)}
\end{array}\right)
$$

where

$$
\star=\left\{\begin{array}{l}
\frac{q_{2}^{\left(m_{2}\right)}\left(1, \mu^{*}\right) p_{2}^{\left(n_{2}-1\right)}\left(1, \mu^{*}\right)-q_{2}^{\left(m_{2}-1\right)}\left(1, \mu^{*}\right) p_{2}^{\left(n_{2}\right)}\left(1, \mu^{*}\right)}{\left[q_{2}^{\left(m_{2}\right)}\left(1, \mu^{*}\right)\right]^{2}}- \\
s \frac{q_{1}^{\left(m_{1}\right)}\left(1, \mu^{*}\right) p_{1}^{\left(n_{1}-1\right)}\left(1, \mu^{*}\right)-q_{1}^{\left(m_{1}-1\right)}\left(1, \mu^{*}\right) p_{1}^{\left(n_{1}\right)}\left(1, \mu^{*}\right)}{\left[q_{1}^{\left(m_{1}\right)}\left(1, \mu^{*}\right)\right]^{2}}, \quad \text { if } \quad \delta_{1}=\delta_{2}=1 \\
\frac{q_{2}^{\left(m_{2}\right)}\left(1, \mu^{*}\right) p_{2}^{\left(n_{2}-1\right)}\left(1, \mu^{*}\right)-q_{2}^{\left(m_{2}-1\right)}\left(1, \mu^{*}\right) p_{2}^{\left(n_{2}\right)}\left(1, \mu^{*}\right)}{\left[q_{2}^{\left(m_{2}\right)}\left(1, \mu^{*}\right)\right]^{2}}- \\
s \frac{p_{1}^{\left(n_{1}\right)}\left(1, \mu^{*}\right)}{q_{1}^{\left(m_{1}\right)}\left(1, \mu^{*}\right)}, \quad \text { if } \quad \delta_{2}=1 \text { and } m=n_{1}-m_{1}+1 \\
\frac{q_{2}^{\left(m_{2}\right)}\left(1, \mu^{*}\right) p_{2}^{\left(n_{2}-1\right)}\left(1, \mu^{*}\right)-q_{2}^{\left(m_{2}-1\right)}\left(1, \mu^{*}\right) p_{2}^{\left(n_{2}\right)}\left(1, \mu^{*}\right)}{\left[q_{2}^{\left(m_{2}\right)}\left(1, \mu^{*}\right)\right]^{2}}, \\
\text { if } \delta_{2}=1 \text { and } m n_{1}-m_{1}+1 \\
\frac{p_{1}^{\left(n_{2}\right)}\left(1, \mu^{*}\right)}{q_{2}^{\left(m_{2}\right)}\left(1, \mu^{*}\right)}-s \frac{q_{1}^{\left(m_{1}\right)}\left(1, \mu^{*}\right) p_{1}^{\left(n_{1}-1\right)}\left(1, \mu^{*}\right)-q_{1}^{\left(m_{1}-1\right)}\left(1, \mu^{*}\right)}{\left[q_{1}^{\left(m_{1}\right)}\left(1, \mu^{*}\right)\right]^{2}}, \\
\text { if } \quad m=n_{2}-m_{2}+1 \text { and } \delta_{1}=1 \\
-s \frac{q_{1}^{\left(m_{1}\right)}\left(1, \mu^{*}\right) p_{1}^{\left(n_{1}-1\right)}\left(1, \mu^{*}\right)-q_{1}^{\left(m_{1}-1\right)}\left(1, \mu^{*}\right) p_{1}^{\left(n_{1}\right)}\left(1, \mu^{*}\right)}{\left[q_{1}^{\left(m_{1}\right)}\left(1, \mu^{*}\right)\right]^{2}} \\
\text { if } m>n_{2}-m_{2}+1 \text { and } \delta_{1}=1 .
\end{array}\right.
$$


The equation (5.6) on $U_{2}$ is equivalent to

$$
G(\mu)=\delta_{1} \frac{p_{1}^{\left(n_{1}\right)}(\mu, 1)}{q_{1}^{\left(m_{1}\right)}(\mu, 1)}-\delta_{2} \mu \frac{p_{2}^{\left(n_{2}\right)}(\mu, 1)}{q_{2}^{\left(m_{2}\right)}(\mu, 1)}=0 .
$$

The linearized system associated to the vector field (5.9) through the equilibrium solution $\left(\mu^{*}, 0\right)$ becomes

$$
B=\left(\begin{array}{cc}
\frac{d G\left(\mu^{*}\right)}{d \mu} & \star \star \\
0 & -\left[m+1-\left(n_{2}-m_{2}\right)\right] \delta_{2} \frac{p_{2}^{\left(n_{2}\right)}\left(\mu^{*}, 1\right)}{q_{2}^{\left(m_{2}\right)}\left(\mu^{*}, 1\right)}=0
\end{array}\right)
$$

where

$$
\begin{aligned}
& \left\{\begin{array}{l}
\frac{q_{1}^{\left(m_{1}\right)}\left(\mu^{*}, 1\right) p_{1}^{\left(n_{1}-1\right)}\left(\mu^{*}, 1\right)-q_{1}^{\left(m_{1}-1\right)}\left(\mu^{*}, 1\right) p_{1}^{\left(n_{1}\right)}\left(\mu^{*}, 1\right)}{\left[q_{1}^{\left(m_{1}\right)}\left(\mu^{*}, 1\right)\right]^{2}}- \\
s \frac{q_{2}^{\left(m_{2}\right)}\left(\mu^{*}, 1\right) p_{2}^{\left(n_{2}-1\right)}\left(\mu^{*}, 1\right)-q_{2}^{\left(m_{2}-1\right)}\left(\mu^{*}, 1\right) p_{2}^{\left(n_{2}\right)}\left(\mu^{*}, 1\right)}{\left[q_{2}^{\left(m_{2}\right)}\left(\mu^{*}, 1\right)\right]^{2}}, \quad \text { if } \quad \delta_{1}=\delta_{2}=1 \\
\frac{q_{1}^{\left(m_{1}\right)}\left(\mu^{*}, 1\right) p_{1}^{\left(n_{1}-1\right)}\left(\mu^{*}, 1\right)-q_{1}^{\left(m_{1}-1\right)}\left(\mu^{*}, 1\right) p_{1}^{\left(n_{1}\right)}\left(\mu^{*}, 1\right)}{\left[q_{1}^{\left(m_{1}\right)}\left(\mu^{*}, 1\right)\right]^{2}}- \\
{ }_{\left(n_{2}\right)}
\end{array}\right. \\
& s \frac{p_{2}^{\left(n_{2}\right)}(\mu, 1)}{q_{2}^{\left(m_{2}\right.}\left(\mu^{*}, 1\right)}, \quad \text { if } \quad \delta_{1}=1 \text { and } m=n_{2}-m_{2}+1 \\
& \star \star=\left\{\begin{array}{l}
\frac{q_{2}^{\left(m_{1}\right)}\left(\mu^{*}, 1\right) p_{1}^{\left(n_{1}-1\right)}\left(\mu^{*}, 1\right)-q_{1}^{\left(m_{1}-1\right)}\left(\mu^{*}, 1\right) p_{1}^{\left(n_{1}\right)}\left(\mu^{*}, 1\right)}{\left[q_{1}^{\left(m_{1}\right)}\left(\mu^{*}, 1\right)\right]^{2}}, \\
\delta_{1}=1 m^{2}>n_{2}-m_{2}+1
\end{array}\right. \\
& \text { if } \quad \delta_{1}=1 \text { and } m>n_{2}-m_{2}+1 \\
& \frac{p_{2}^{\left(n_{1}\right)}\left(\mu^{*}, 1\right)}{q_{1}^{\left(m_{2}\right)}\left(\mu^{*}, 1\right)}-s \frac{q_{2}^{\left(m_{2}\right)}\left(\mu^{*}, 1\right) p_{2}^{\left(n_{2}-1\right)}\left(\mu^{*}, 1\right)-q_{2}^{\left(m_{2}-1\right)}\left(\mu^{*}, 1\right)}{\left[q_{2}^{\left(m_{2}\right)}\left(\mu^{*}, 1\right)\right]^{2}}, \\
& \text { if } m=n_{1}-m_{1}+1 \text { and } \delta_{2}=1 \\
& -s \frac{q_{2}^{\left(m_{2}\right)}\left(\mu^{*}, 1\right) p_{2}^{\left(n_{2}-1\right)}\left(\mu^{*}, 1\right)-q_{2}^{\left(m_{2}-1\right)}\left(\mu^{*}, 1\right) p_{2}^{\left(n_{2}\right)}\left(\mu^{*}, 1\right)}{\left[q_{2}^{\left(m_{2}\right)}\left(\mu^{*}, 1\right)\right]^{2}}, \\
& \text { if } \quad m>n_{1}-m_{1}+1 \text { and } \delta_{2}=1 \text {. }
\end{aligned}
$$

Proposition 1. Let $X$ be a rational vector field, then all the equilibrium solutions of $P(X)$ in $S^{1}$ are hyperbolic, except in the following cases:

- (i) $F(\mu)$ or $G(\mu)$ have multiple real zeros,

- (ii) $\delta_{1}=0$ or $\delta_{2}=0$,

- (iii) $F(\mu)$ or $G(\mu)$ have simple real zeros, $\delta_{1}=\delta_{2}=1$ but $\frac{p_{1}^{\left(n_{1}\right)}\left(1, \mu^{*}\right)}{q_{1}^{\left(m_{1}\right)}\left(1, \mu^{*}\right)}=$ 0 or $\frac{p_{2}^{n_{2}}\left(\mu^{*}, 1\right)}{q_{2}^{\left(m_{2}\right)}\left(\mu^{*}, 1\right)}=0$ for some real $\mu^{*}$ where $\left(\mu^{*}, 0\right)$ is one of the equilibrium solutions. 
Remarks. 1) If $\left(\mu^{*}, 0\right)$ is an equilibrium on $U_{1}$ and the condition $\frac{p_{1}^{\left(n_{1}\right)}\left(1, \mu^{*}\right)}{q_{1}^{\left(m_{1}\right)}\left(1, \mu^{*}\right)}=$ 0 is satisfied then, by equation (5.7), we have that $\frac{p_{2}^{\left(n_{2}\right)}\left(1, \mu^{*}\right)}{q_{2}^{\left(m_{2}\right)}\left(1, \mu^{*}\right)}=0$. Analogously, if $\left(\mu^{*}, 0\right)$ is an equilibrium on $U_{2}$ and the condition $\frac{p_{2}^{\left(n_{2}\right)}\left(\mu^{*}, 1\right)}{q_{2}^{\left(m_{2}\right)}\left(\mu^{*}, 1\right)}=0$ is satisfied then, by equation (5.9), we have that $\frac{p_{1}^{\left(n_{1}\right)}\left(\mu^{*}, 1\right)}{q_{1}^{\left(m_{1}\right)}\left(\mu^{*}, 1\right)}=0$.

2) If neither $F(\mu)$ nor $G(\mu)$ have real zeros, and $P(X)$ is smooth on $S^{1}$ then the equator is a closed orbit. If $P(X)$ has a finite number of singularities then the integral curves of $P(X)$ is a finite union of arc of curves on $S^{1}$.

3) If $\delta_{1}=\delta_{2}=1$ then $n_{1}-m_{1}=m=n_{2}=m_{2}$ and supposing that $F(\mu)$ and $G(\mu)$ do not have real zeros, then $n_{1}+m_{2}=n_{2}+m_{1}$ is odd. To prove it, we write $F(\mu)$ and $G(\mu)$ as:

$$
F(\mu)=\frac{Q_{n}(1, \mu)-\mu P_{n}(1, \mu)}{q_{1}^{\left(m_{1}\right)}(1, \mu) q_{2}^{\left(m_{2}\right)}(1, \mu)}
$$

and

$$
G(\mu)=\frac{P_{n}(\mu, 1)-\mu Q_{n}(\mu, 1)}{q_{1}^{\left(m_{1}\right)}(\mu, 1) q_{2}^{\left(m_{2}\right)}(\mu, 1)}
$$

where

$$
Q_{n}(x, y)=p_{2}^{\left(n_{2}\right)}(x, y) q_{1}^{\left(m_{1}\right)}(x, y)
$$

and

$$
P_{n}(x, y)=p_{1}^{\left(n_{1}\right)}(x, y) q_{2}^{\left(m_{2}\right)}(x, y) .
$$

Supposing that $n$ is even, then

$$
x Q_{n}(x, y)-y P_{n}(x, y)
$$

is odd and will have a zero either for $x=0$ or for some finite value of $y / x$. In both cases we would have a singularity at infinity, contrary to the assumption.

Proposition 2. One hyperbolic equilibrium solution $\left(\mu^{*}, 0\right)$ in $U_{1}$ (resp. in $U_{2}$ ) of (3.1) at the infinity can be a: 
- saddle point if $\frac{d F\left(\mu^{*}\right)}{d \mu} \frac{p_{1}^{\left(n_{1}\right)}\left(1, \mu^{*}\right)}{q_{1}^{\left(m_{1}\right)}\left(1, \mu^{*}\right)}<0$ (resp. $\left.\frac{d G\left(\mu^{*}\right)}{d \mu} \frac{p_{2}^{n_{2}}\left(\mu^{*}, 1\right)}{q_{2}^{\left(m_{2}\right)}\left(\mu^{*}, 1\right)}<0\right)$;

- stable node if $\frac{d F\left(\mu^{*}\right)}{d \mu}<0$ and $\frac{p_{1}^{\left(n_{1}\right)}\left(1, \mu^{*}\right)}{q_{1}^{\left(m_{1}\right)}\left(1, \mu^{*}\right)}<0$ (resp. $\frac{d G\left(\mu^{*}\right)}{d \mu}<0$ and $\left.\frac{p_{2}^{n_{2}}\left(\mu^{*}, 1\right)}{q_{2}^{\left(m_{2}\right)}\left(\mu^{*}, 1\right)}<0\right)$

- unstable node if $\frac{d F\left(\mu^{*}\right)}{d \mu}>0$ and $\frac{p_{1}^{\left(n_{1}\right)}\left(1, \mu^{*}\right)}{q_{1}^{\left(m_{1}\right)}\left(1, \mu^{*}\right)}>0$ (resp. $\frac{d G\left(\mu^{*}\right)}{d \mu}>0$ and $\left.\frac{p_{2}^{n_{2}}\left(\mu^{*}, 1\right)}{q_{2}^{\left(m_{2}\right)}\left(\mu^{*}, 1\right)}>0\right)$.

\subsection{Equilibrium solutions for quotient of powers of polynomial vector fields}

Now, we will analyze the vector field given by (3.7) satisfying (3.2) for $n=2$, i.e., the vector field $X$ in $(2.1)$ on $\mathbb{R}^{2}$ is given by the quotient of power of polynomial vector fields. Maintaining the preliminary notations, the equation (5.5) in $U_{1}$ became equivalent to

$$
H(\mu)=\delta_{2} \frac{\left[p_{2}^{\left(n_{2}\right)}(1, \mu)\right]^{\alpha_{2}}}{\left[q_{2}^{\left(m_{2}\right)}(1, \mu)\right]^{\beta_{2}}}-\delta_{1} \mu \frac{\left[p_{1}^{\left(n_{1}\right)}(1, \mu)\right]^{\alpha_{1}}}{\left[q_{1}^{\left(m_{1}\right)}(1, \mu)\right]^{\beta_{1}}}=0 .
$$

To analyze the hyperbolicity of the equilibrium solutions it is necessary to calculate the variational equations of the vector field (5.2) through the equilibrium solution $\left(\mu^{*}, 0\right)$. Therefore, the linear part is given by

$(5.12) C=\left(\begin{array}{cc}\frac{d H\left(\mu^{*}\right)}{d \mu} & \otimes \\ 0 & -\left[m+1-\left(\alpha_{1} n_{1}-\beta_{1} m_{1}\right)\right] \delta_{1} \frac{\left[p_{1}^{\left(n_{1}\right)}\left(1, \mu^{*}\right)\right]^{\alpha_{1}}}{\left[q_{1}^{\left(m_{1}\right)}\left(1, \mu^{*}\right)\right]^{\beta_{1}}}\end{array}\right)$

where $\otimes$ is calculated in a similar way as in the rational case.

The equation (5.6) on $U_{2}$ is equivalent to

$$
K(\mu)=\delta_{1} \frac{\left[p_{1}^{\left(n_{1}\right)}(\mu, 1)\right]^{\alpha_{1}}}{\left[q_{1}^{\left(m_{1}\right)}(\mu, 1)\right]^{\beta_{1}}}-\delta_{2} \mu \frac{\left[p_{2}^{\left(n_{2}\right)}(\mu, 1)\right]^{\alpha_{2}}}{\left[q_{2}^{\left(m_{2}\right)}(\mu, 1)\right]^{\beta_{2}}}=0 .
$$

Thus, the linear part associated to the vector field (5.3) through the equilibrium solution $\left(\mu^{*}, 0\right)$ is 
(5.14) $D=\left(\begin{array}{cc}\frac{d K\left(\mu^{*}\right)}{d \mu} & \otimes \otimes \\ 0 & -\left[m+1-\left(\alpha_{2} n_{2}-\beta_{2} m_{2}\right)\right] \delta_{2} \frac{\left[p_{2}^{\left(n_{2}\right)}\left(\mu^{*}, 1\right)\right]^{\alpha_{2}}}{\left[q_{2}^{\left(m_{2}\right)}\left(\mu^{*}, 1\right)\right]^{\beta_{2}}}\end{array}\right)$

where $\otimes \otimes$ is calculated in a similar way than in the rational case.

Proposition 3. Let $X$ be a quotient of powers of polynomial vector fields, then all the equilibrium solutions of $P(X)$ in $S^{1}$ are hyperbolic, except in the following cases:

- (i) $H(\mu)$ or $K(\mu)$ have multiple real zeros,

- (ii) $\delta_{1}=0$ or $\delta_{2}=0$,

- (iii) $H(\mu)$ or $K(\mu)$ have simple real zeros, $\delta_{1}=\delta_{2}=1$ but $\frac{\left[p_{1}^{\left(n_{1}\right)}\left(1, \mu^{*}\right)\right]^{\alpha_{1}}}{\left[q_{1}^{\left(m_{1}\right)}\left(1, \mu^{*}\right)\right]^{\beta_{1}}}=0$ or $\frac{\left[p_{2}^{\left(n_{2}\right)}\left(\mu^{*}, 1\right)\right]^{\alpha_{2}}}{\left[q_{2}^{\left(m_{2}\right)}\left(\mu^{*}, 1\right)\right]^{\beta_{2}}}=0$ for some real $\mu^{*}$, where $\left(\mu^{*}, 0\right)$ is one of the equilibrium solutions.

The character of the stability is described in the following proposition.

Proposition 4. One hyperbolic equilibrium solution $\left(\mu^{*}, 0\right)$ in $U_{1}$ (resp. in $U_{2}$ ) of (3.7) at the infinity can be a:

- saddle point if $\frac{d H\left(\mu^{*}\right)}{d \mu} \frac{\left[p_{1}^{\left(n_{1}\right)}\left(1, \mu^{*}\right)\right]^{\alpha_{1}}}{\left[q_{1}^{\left(m_{1}\right)}\left(1, \mu^{*}\right)\right]^{\beta_{1}}}<0$ (resp. $\frac{d K\left(\mu^{*}\right)}{d \mu} \frac{\left[p_{2}^{n_{2}}\left(\mu^{*}, 1\right)\right]^{\alpha_{2}}}{\left[q_{2}^{\left(m_{2}\right)}\left(\mu^{*}, 1\right)\right]^{\beta_{2}}}<$ $0)$;

- stable node if $\frac{d H\left(\mu^{*}\right)}{d \mu}<0$ and $\frac{\left[p_{1}^{\left(n_{1}\right)}\left(1, \mu^{*}\right)\right]^{\alpha_{1}}}{\left[q_{1}^{\left(m_{1}\right)}\left(1, \mu^{*}\right)\right]^{\beta_{1}}}<0$ (resp. $\frac{d K\left(\mu^{*}\right)}{d \mu}<0$ and $\left.\frac{\left[p_{2}^{n_{2}}\left(\mu^{*}, 1\right)\right]^{\alpha_{2}}}{\left[q_{2}^{\left(m_{2}\right)}\left(\mu^{*}, 1\right)\right]^{\beta_{2}}}<0\right)$

- unstable node if $\frac{d H\left(\mu^{*}\right)}{d \mu}>0$ and $\frac{\left[p_{1}^{\left(n_{1}\right)}\left(1, \mu^{*}\right)\right]^{\alpha_{1}}}{\left[q_{1}^{\left(m_{1}\right)}\left(1, \mu^{*}\right)\right]^{\beta_{1}}}>0$ (resp. $\frac{d K\left(\mu^{*}\right)}{d \mu}>0$ and $\left.\frac{\left[p_{2}^{n_{2}}\left(\mu^{*}, 1\right)\right]^{\alpha_{2}}}{\left[q_{2}^{\left(m_{2}\right)}\left(\mu^{*}, 1\right)\right]^{\beta_{2}}}>0\right)$. 


\section{Application to Celestial Mechanic}

In [3] and [4] we can find very nice applications of the Poincaré compactification vector field. In fact, they studied the bounded part using the Wang's coordinates (see [11]) but, for studying the unbounded part they used the Poincare's compactification. In both papers the authors used the Poincaré compactification but they did not justify all the arguments involved in its applications.

Studying the qualitative dynamic of the flow in both the bounded and unbounded part it is possible to understand the global flow in these problems. In [3] the authors studied the parabolic non collision restricted three body problem, where the mass points, of equal masses $m_{1}=m_{2}>0$, moving under Newton's law of attraction, in a non-collision parabolic orbit, while their center of mass is at rest and it is consider the third mass point, of mass $m_{3}=0$, moving on the straight line $L$ perpendicular to the plane of motion of the first two mass points and passing through their center of mass. If $(x / 2, y / 2,0)$ denotes the position of the mass point $m_{1}$ and $(0,0, z)$ the position of $m_{3}$, then the equations of motion for $x, y, z$ are

$$
\begin{aligned}
& \ddot{x}=-\frac{2 x}{\left(x^{2}+y^{2}\right)^{3 / 2}} \\
& \ddot{y}=-\frac{2 y}{\left(x^{2}+y^{2}\right)^{3 / 2}} \\
& \ddot{z}=-\frac{16 z}{\left(x^{2}+y^{2}+4 z^{2}\right)^{3 / 2}} .
\end{aligned}
$$

In [4] the author studied the parabolic collision restricted three body problem which is a similar problem but in this case it is possible to have collision. Let $x$ denotes the distance between the two primaries $m_{1}$ and $m_{2}$. Thus, $(x / 2,0)$ denotes the position of $m_{1}$ and consequently $(-x / 2,0)$ that of $m_{2}$. If we denote by $(0, y)$ the position of $m_{3}$, then the equations of motion for $x$ and $y$ are

$$
\begin{aligned}
& \ddot{x}=-\frac{2}{x^{2}} 16 y \\
& \ddot{y}=-\frac{16 y}{\left(x^{2}+4 y^{2}\right)^{3 / 2}} .
\end{aligned}
$$

Using Wang's coordinates both problems have the following vector field

$$
X=X\left(x_{1}, x_{2}\right)=\left(-\frac{x_{1}}{4}+x_{2}, \frac{x_{2}}{8}-\frac{2 x_{1}}{\left(4+x_{1}^{2}\right)^{3 / 2}}\right) .
$$

This vector field is like the vector field defined in (3.10), with

$$
X_{H}=\left(-\frac{x_{1}}{4}+x_{2}, \frac{x_{2}}{8}\right), \quad \text { and } \quad X_{F}=\left(0,-\frac{2 x_{1}}{\left(4+x_{1}^{2}\right)^{3 / 2}}\right)
$$


being $k_{1}=k_{2}=1, n_{1}=n_{2}=0, m_{1}=0, m_{2}=2, \quad \alpha_{1}=0$ and $\beta_{1}=3 / 2$. Therefore, in this case, applying Corollary 1 , we have that $m=1$. In order to represent the flow of $P(X)$ on $S^{1}$ we use its projection on the closure of the north hemisphere with respect to the $y_{3}$-axis on the plane $\left(y_{1}, y_{2}\right)$, that is, its projection on the disc $\left\{\mathbf{y} \in \mathbb{R}^{3} / y_{1}^{2}+y_{2}^{2} \leq 1\right.$ and $\left.y_{3}=0\right\}$, called the Poincaré disc. The interior of the Poincaré disc represents the flow in the finite part of system (6.1) or (6.2) and the boundary $S^{1}$ of the Poincaré disc, represents the infinity part of system (6.1) or (6.2). The following picture describe the global flow at finite and infinite part of system (6.1) or (6.2) on the Poincaré disc when $0<t<+\infty$.

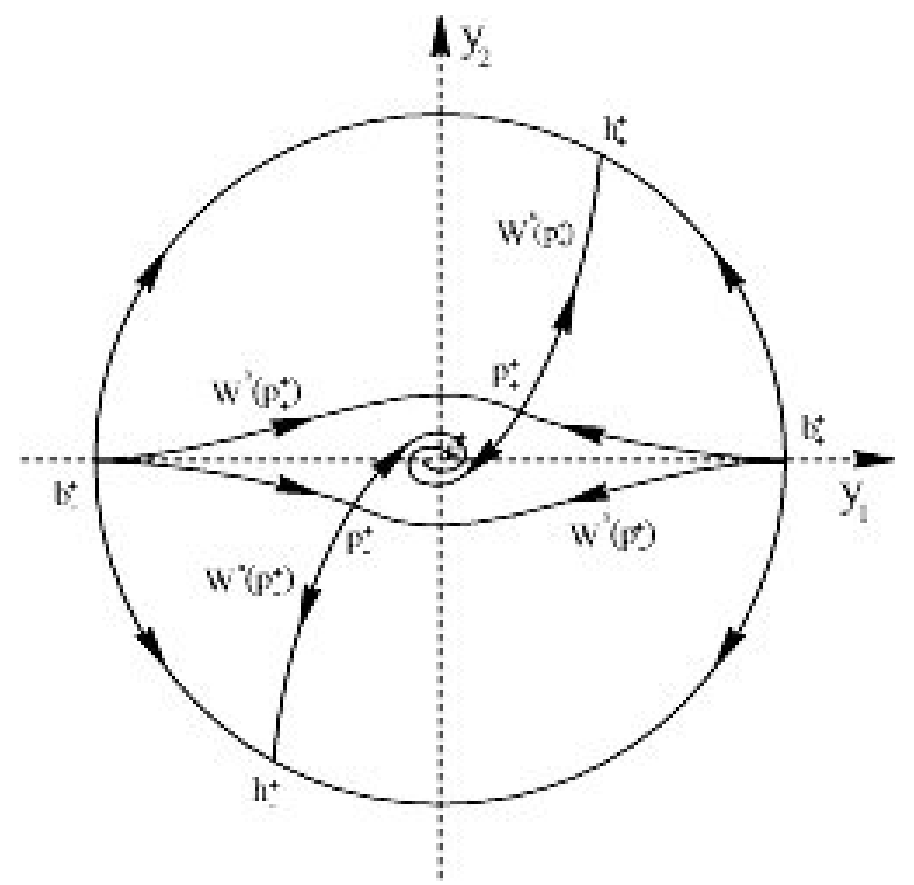

Figure 2 : The global flow when $0<t<+\infty$.

We have three equilibrium solutions in the finite part: one stable focus and two saddle points; and four equilibrium solutions in the infinite part: two stable nodes and two unstable nodes.

Acknowledgement. We wish to thank the refereee and to Hildeberto Cabral (UFPE-Brazil) for their useful suggestions and comments. 


\section{References}

[1] Chazy, J. : Sur l'allure du mouvement dans le problème des trois corps quand le temps croit indèfiniment. Ann. Ecole Norm. Sup. 39 (3), pp. 29-130, (1922).

[2] Cima, A., and Llibre, J. :. Bounded polynomial vector fields. Trans. Amer. Math. Soc. 318, pp. 557-579, (1990).

[3] Cors, J., and Llibre, J. : The global flow of the parabolic restricted three body problem. Phd thesis, Universitat Autònoma de Barcelona, (1994).

[4] Cors, J., and Llibre, J. : Qualitative study of the parabolic collision restricted three body problem. Contemporary Mathematics. 198, pp. 1-19, (1996).

[5] Delgado, J. ; Lacomba, E. A.; Llibre, J.; Pérez, E. : Poincaré compactification of the Kepler and the collinear three body problem. Seminar on Dynamical Systems (St. Petersburg, 1991), pp. 117-128, Progr. Nonlinear Differential Equations Appl., 12, Birkhäuser, Basel, (1994).

[6] Delgado, J. ; Lacomba, E. A.; Llibre, J.; Pérez, E. : Poincaré compactification of the collinear three body problem. Hamiltonian systems and celestial mechanics (Guanajuato, 1991), 85-100, Adv. Ser. Nonlinear Dynam., 4, World Sci. Publishing, River Edge, NJ, (1993).

[7] Delgado, J. ; Lacomba, E. A. ; Llibre, J. ; Pérez, E. : Poincaré compactification of Hamiltonian polynomial vector fields. Hamiltonian dynamical systems (Cincinnati, OH, 1992), 99-114, IMA Vol. Math. Appl., 63, Springer, New York, (1995).

[8] González, E.: Generic properties of polynomial vector fields at infinity. Trans. Amer. Math. Soc. 143, pp. 201-222, (1969).

[9] Hegie, D. : A global regularization of the gravitational $n$-body problem. Cel. Mech. 10, pp. 217-241, (1974). 
[10] Poincaré, H. : Mémoire sur les courbes définies par une equation différentielle. J. Mathématiques (3), 7, pp. 375-422, (1881).

[11] Wang, Q. : Qualitative study of n-body problem: Untized momentum transformation and its application restricted isoceles three-body problem with positive energy, Space Dynamics and Celestial Mechanics, K. B. Bhantnagar, ed., pp. 61-69, (1986).

Received : November 2002.

\section{Claudio Vidal}

Departamento de Matemática

Universidade Federal de Pernambuco

Av. Prof. Luiz Freire, s/n

Cidade Universitária

Recife-PE

Brasil

e-mail : claudio@dmat.ufpe.br

and

\section{Pedro Gómez}

Departamento de Matemática

Universidade Federal da Paraiba

Cidade Universitária, João Pessoa-PB

Brazil

e-mail : venegas@mat.ufpb.br 